\title{
Nasal Airflow Rate Affects the Sensitivity and Pattern of Glomerular Odorant Responses in the Mouse Olfactory Bulb
}

\author{
Yuki Oka, Yoshiki Takai, and Kazushige Touhara \\ Department of Integrated Biosciences, The University of Tokyo, Chiba 277-8562, Japan
}

\begin{abstract}
Sniffing is a characteristic odor sampling behavior in various mammalian species, which is associated with an increase in both nasal airflow rate and breathing frequency. Although the importance of sniffing in olfaction is well recognized, it has been challenging to separate the effect of airflow rate and sniffing frequency in vivo. In this study, we examined the individual effects of airflow rate and frequency on odorant responses of glomeruli in the mouse olfactory bulb $(\mathrm{OB})$ using calcium imaging techniques and an artificial sniffing system. We found that nasal airflow rate, but not sniffing frequency, affected the apparent glomerular responses. When measured using OB imaging, apparent sensitivity for some of the odorants was significantly greater at the high nasal flow rates, while other odorants exhibited the opposite effect. In a single defined glomerulus, the sensitivity shift caused by changes in flow rate varied between odorants, suggesting that the flow rate effect is dependent on the chemical properties of an odorant rather than on the specific characteristics of the expressed olfactory receptor. Using natural flavors containing a variety of odorants, different glomerular activation patterns were observed between breathing and sniffing condition, likely due to odorant-dependent flow rate effects. Our results provide important information on in vivo odorant recognition and suggest that odor representation in the $\mathrm{OB}$ is not fixed but rather varies significantly depending on the respiratory state.
\end{abstract}

\section{Introduction}

Odorants are recognized by peripheral olfactory sensory neurons (OSNs) in the olfactory epithelium, where hundreds of olfactory receptors (ORs) mediate odorant signal transduction (Mombaerts, 2004; Touhara and Vosshall, 2009). Due to the singular OR expression and neural projection patterns, the glomerular activation patterns in the olfactory bulb $(\mathrm{OB})$ is believed to represents the odorant quality (Mombaerts, 2006; Mori et al., 2006; Touhara, 2007). To ensure precise olfactory detection and perception, efficient delivery mechanism of odorant molecules to the OR site is required in the nasal cavity. This initial delivery event is commonly divided into two steps: (1) odorant inhalation into the nasal cavity, and (2) dissolution of odorants into the aqueous mucosal layer.

Odorant sampling behavior allows for the efficient absorption of odorants into the mucosal layer to activate ORs. In vitro studies have revealed that an OR exhibits a relatively low odorant affinity, with an $\mathrm{EC}_{50}$ value typically between $10^{-6}$ and $10^{-3} \mathrm{M}$ in aqueous solution (Oka et al., 2004; Katada et al., 2005; Abaffy et al., 2006; Yoshikawa and Touhara, 2009). In contrast, animals are able to behaviorally detect much lower concentrations of odorants, generally ranging between $10^{-10}$ and $10^{-7} \mathrm{M}$ in the air phase (Doty,

\footnotetext{
Received March 25, 2009; revised July 18, 2009; accepted Aug. 12, 2009.

This work was supported in part by a grant from the Program for Promotion of Basic Research Activities for Innovative Biosciences, Japan (PROBRAIN), a Grant-in-Aid for Scientific Research on Priority Areas (to K.T.), and a Grant-in-Aid for JSPS Fellows (to Y.0.). We thank T. Hasegawa Co. Ltd. for odorants.

Correspondence should be addressed to Kazushige Touhara at the above address. E-mail: touhara@k.u-tokyo.ac.jp.

Y. Oka's present address: Columbia University, New York, NY 10032.

DOI:10.1523/JNEUROSCI.1415-09.2009

Copyright $\odot 2009$ Society for Neuroscience $\quad 0270-6474 / 09 / 2912070-09 \$ 15.00 / 0$
}

1994; Cometto-Muñiz and Abraham, 2008). These differences suggest that odorants are efficiently concentrated into the mucus layers during the odor sampling process. Indeed, an $\sim 1000$-fold difference in the apparent sensitivity has been observed between responsiveness of an OR in vitro and of an OR-defined glomerulus in vivo (Oka et al., 2006). These results indicate that there is a factor increasing perceived odorant sensitivity under the physiological condition.

Odorants are inhaled into the nasal cavity under different airflow rates and frequency, which is tightly regulated by the breathing condition (Kepecs et al., 2006; Scott, 2006). Sniffing is a phase of respiration that is defined by a fast respiratory frequency and an increased nasal airflow, and is an action frequently observed in various mammalian species (Halpern, 1983). Odor identification tests have suggested that perception sensitivity is significantly increased upon sniffing behavior (Rehn, 1978; Teghtsoonian and Teghtsoonian, 1984), demonstrating that sniffing is an important regulator of the efficiency at which odorants are absorbed into the mucus layer, and affects the apparent odorant sensitivity in the olfactory system. In mice, respiratory frequency increases from $2-5 \mathrm{~Hz}$ under normal breathing conditions to 4-12 $\mathrm{Hz}$ when sniffing, while the nasal airflow rate changes from 10-20 $\mathrm{ml} / \mathrm{min}$ to $50-100 \mathrm{ml} / \mathrm{min}$, respectively (Youngentob et al., 1987; Wesson et al., 2008). These two factors may be regulated separately in vivo, since a previous study has revealed that animals perform different numbers of sniffs to different odors without affecting nasal airflow rate (Laing, 1982). Thus, it is possible that vertebrate olfactory performance is differentially affected by each factor involved in sniffing behavior.

To examine the parameters of sniffing behavior that control odorant sensitivity in vivo, we developed a technique that allows 

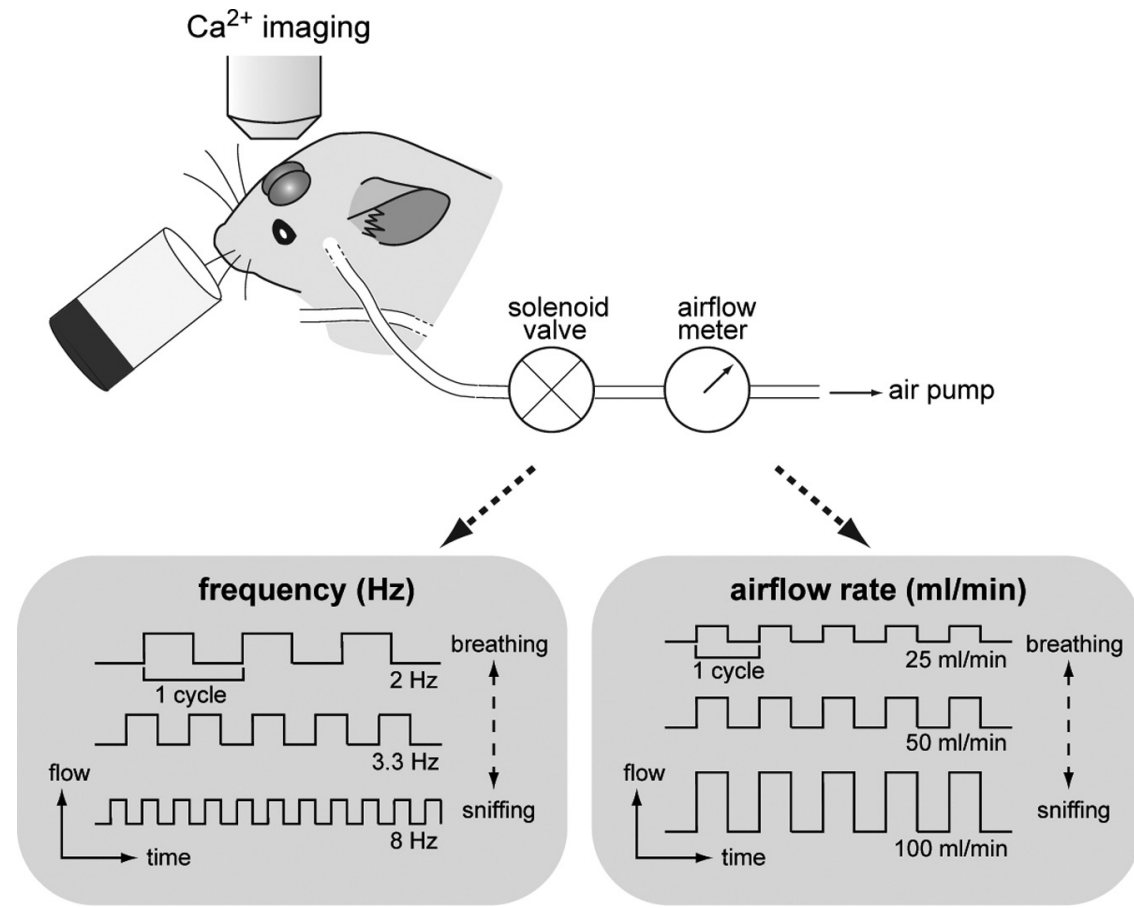

Figure 1. Schematic diagram of the sniffing control system. The upper tube was connected to the solenoid valve and airflow meter, which control sniffing frequency and airflow rate, respectively. The lower tube was directly jointed to the lower trachea and remained open for normal breathing. In our experiments, frequency and airflow rate were altered independently and in a stepwise manner from 2 to $8 \mathrm{~Hz}$ (for frequency) and from 25 to $100 \mathrm{ml} / \mathrm{min}$ (for airflow rate).

for examining the effects of nasal flow rate and respiratory frequency individually on odorant responses in the OB. Combining calcium imaging and an artificial sniffing system, we show that nasal airflow rate, but not respiratory frequency, is a key factor that regulates olfactory sensitivity of the glomeruli. Furthermore, the flow rate effect is obvious for natural flavors in that the activation pattern in the $\mathrm{OB}$ is different between fast and slow airflow rate. The current results highlight a role for sniffing in the regulation of olfactory sensitivity, and also partly account for our experience that odorant intensity and quality are perceived differently depending on respiratory conditions.

\section{Materials and Methods}

Odorants. The odorants used in the current study were kindly provided by T. Hasegawa Co. and Takasago Co. or purchased from Sigma. The purity of odorants was checked using thin-layer chromatography or gas chromatography-mass spectrometry (GC-MS). Odorants were prepared in $5 \mathrm{ml}$ glass tubes, either as a pure substance or diluted in mineral oil. As the theoretical vapor concentration often varies greatly from the actual concentration, we directly measured the vapor concentration of each odorant, both pure substance and diluted form, using GC-MS (Shimadzu) as described in supplemental Figure S1, available at www. jneurosci.org as supplemental material. Natural flavor oil was directly diluted in mineral oil to indicated concentrations.

Recording of odorant response in the $\mathrm{OB}$. For $\mathrm{Ca}^{2+}$ imaging, we loaded dextran-conjugated calcium dye into OSNs as described previously (Wachowiak and Cohen, 2001; Oka et al., 2006). Briefly, a $1.5 \mu \mathrm{l}$ of $6 \%$ Calcium Green-1 dextran 3 kDa (Molecular Probes, Eugene) in $\mathrm{Ca}^{2+}$ free Ringer's solution was perfused into the nasal cavity using a shortened gel-loading tip under ketamine $(90 \mathrm{~g} / \mathrm{kg})$ and xylazine $(10 \mathrm{~g} / \mathrm{kg})$ anesthesia. Perfusion was repeated at $3 \mathrm{~min}$ intervals until a total volume of $6-8$ $\mu \mathrm{l}$ was injected. This volume was sufficient to load the OSNs that innervate the entire dorsal portion of the $\mathrm{OB}$ with enough fluorescent dye to provide a strong signal. 3-7 d after the dye loading, mice were then anesthetized with a subcutaneous injection of ketamine $(70 \mathrm{mg} / \mathrm{kg})$ and medetomidine $(0.5 \mathrm{mg} / \mathrm{kg})$. Using a dental drill, the skull overlying the dorsal surface of the $\mathrm{OB}$ was thinned and removed, leaving the dura intact. The exposed area was then filled with $1.5 \%$ agarose gel and enclosed with a cover glass. Under normal breathing conditions, the breathing rate was maintained between 2.0 and $3.5 \mathrm{~Hz}$ for the duration of the experiment. The $\mathrm{OB}$ was illuminated with $500 \pm 25 \mathrm{~nm}$, and emission $>530 \mathrm{~nm}$ was collected using a $525 \mathrm{~nm}$ dichroic mirror. In mOR-EG transgenic mice that expressed GFP in mOR-EG-positive OSNs (Oka et al., 2006), Calcium Green-1 signal was separated from the GFP signal using a custom-made filter and a $560 \mathrm{~nm}$ dichroic mirror with excitation at $537 \pm 15 \mathrm{~nm}$ and emission at $565 \mathrm{~nm}$. For synapto-pHluorin ( $\mathrm{SpH})$-based imaging (Bozza et al., 2004), the OB was illuminated at $475 \pm 15 \mathrm{~nm}$, and emitted light at wavelengths between 510 and $550 \mathrm{~nm}$ were collected using a $505 \mathrm{~nm}$ dichroic mirror.

In each recording, data were collected for $15 \mathrm{~s}$. Odor stimulation was applied for a duration of $3 \mathrm{~s}$ starting at $5 \mathrm{~s}$. Stimulations were delivered by placing odorant-containing glass tubes within $2 \mathrm{~mm}$ of the nostril. Images were acquired using Aqua Cosmos software and an ORCA2-ER CCD camera (Hamamatsu Photonics, Hamamatsu) with a resolution of $672 \times$ 522 pixels at $12 \mathrm{~Hz}$ (for $\mathrm{Ca}^{2+}$ imaging) or $6 \mathrm{~Hz}$ (for $\mathrm{SpH}$ imaging). The imaging area was $2.3 \times$ $1.4 \mathrm{~mm}$ using a $10 \times$ objective lens and $5.6 \times$ $3.4 \mathrm{~mm}$ using a $4 \times$ objective lens.

We used 4- to 8-week-old C57BL/6 mice or transgenic mice. Mice were bred in a pathogen-free environment, and all experiments were performed in accordance with the guidelines approved by our institution's Animal Experiments Committee.

Artificial sniffing system. A double tracheotomy was performed as described previously (Wachowiak and Cohen, 2001), and an artificial sniffing system was used to control odorant access. This system used the combination of a solenoid valve (Cole Parmer) and a fine airflow meter (KOFLOC). The naris on the side of the Calcium Green-1 injection remained open to maintain a stable airflow. The upper tracheotomy tube was vacuumed with negative pulse pressure for odorant presentation. The airflow rate and sniffing frequency were arbitrarily changed from $25-100 \mathrm{ml} / \mathrm{min}$ and $2-8 \mathrm{~Hz}$, respectively. For an airflow rate of $<25$ $\mathrm{ml} / \mathrm{min}$, we used a peristaltic pump (Peri-Star pro, WPI) to stably generate very low air speeds through the naris.

Data analysis. Data acquired from the $\mathrm{Ca}^{2+}$ and $\mathrm{SpH}$ imaging were analyzed individually and the relative signal amplitude calculated as described previously (Wachowiak and Cohen, 2001). Briefly, the fluorescence level of each pixel at the peak of the response was subtracted from the resting signal intensity recorded just before the stimulus onset, and divided by the resting intensity to adjust for unequal dye labeling or uneven illumination. In the analysis of dose dependence, the $\Delta F / F$ value was quantified and presented as a percentage of the maximum response in each glomerulus.

Spatial maps were constructed from each pixel's signal by subtracting the temporal average of a time window just preceding the stimulus $\left(t_{1}\right)$ from the temporal average centered near the peak of the response $\left(t_{2}\right)$, divided by the temporal average of $t_{1}$. To construct odor maps, we averaged the data between the period from 2.7 to $4.6 \mathrm{~s}$ for $t_{1}$ and from 5.5 to $7.8 \mathrm{~s}$ for $t_{2}$. For the generation of figures, all maps were smoothed using a $3 \times 3$ pixel kernel and normalized to the maximum signal amplitude $(\Delta F / F)$ for the trial. All spatial data were analyzed and processed using Aqua Cosmos software (Hamamatsu Photonics) and Photoshop (Adobe). 


\section{Results}

Sniffing behavior is associated with changes in both airflow rate and breathing frequency (Kepecs et al., 2006). To examine the effects of these two factors on odorant responses under the physiological condition, we developed an artificial sniffing system that directly controls sniffing frequency and airflow rate separately by a solenoid valve and an airflow meter (Fig. 1). Odorant responses were monitored using in vivo $\mathrm{Ca}^{2+}$ imaging in the $\mathrm{OB}$. As each olfactory neuron expresses a single OR stochastically from $\sim 1000$ OR members (Touhara, 2002; Buck, 2004), and that olfactory neurons expressing the same OR are known to innervate the same glomeruli (Mombaerts, 2006), $\mathrm{Ca}^{2+}$ response in a single glomerulus corresponds the activation of a single type of OR. To assess the reliability of our artificial sniffing system, we compared the odorant response of an OR-defined glomerulus under normal breathing conditions with that of an artificially controlled respiration condition. The Ea glomerulus, which is innervated by OSNs expressing the eugenol (EG) receptor MOR224-9 (Oka et al., 2006), resulted in a response to EG with an $\mathrm{EC}_{50}$ value of $5.8 \mathrm{~nm}$ under a freely breathing anesthetized condition (Fig. 2). Under artificially controlled respiratory conditions with a frequency of $3.3 \mathrm{~Hz}$ and a flow rate of $50 \mathrm{ml} / \mathrm{min}$, which reflects normal breathing conditions (Onodera et al., 1997), the Ea glomerulus exhibited similar dose-response properties as that obtained under freely breathing condition (i.e., an $\mathrm{EC}_{50}$ value of $6.5 \mathrm{~nm}(n=3)$ (Fig. 2 ). We did not observe any response in the absence of odorant stimulation (data not shown). These results demonstrated that this artificial sniffing system is able to recapitulate animal respiration.

Using this system, we first examined the effects of respiratory frequency on glomerular responses to EG. The respiratory frequency was altered in a stepwise manner from 2 to $3.3 \mathrm{~Hz}$ and then finally to $8 \mathrm{~Hz}$, as a reflection of slow breathing, normal breathing, and sniffing states, respectively. Airflow rate was fixed at $50 \mathrm{ml} / \mathrm{min}$ for these experiments. We found no significant differences in the apparent sensitivity of EG-responsive glomeruli under these frequency conditions (Fig. $3 A$ ). The $\mathrm{EC}_{50}$ values for an EG response in the Ea glomerulus were $5.9 \mathrm{~nm}$ for $8 \mathrm{~Hz}, 4.3$ $\mathrm{nm}$ for $3.3 \mathrm{~Hz}$ and $5.1 \mathrm{nM}$ for $2 \mathrm{~Hz}$, indicating that the apparent sensitivity is not dependent on respiratory frequency (Fig. $3 B$ ). Similar dose-response curves were obtained when the frequency was altered at any other constant flow rate, and a glomerular response was not observed in the absence of odorant stimulation (data not shown). These results demonstrate that a change in a respiratory frequency exhibits only a minor effect on the apparent sensitivity of glomeruli to EG.

In contrast, changes in airflow rate greatly affected EG responses in the $\mathrm{OB}$ (Fig. 4A). When we altered airflow rate from 25 $\mathrm{ml} / \mathrm{min}$ to $100 \mathrm{ml} / \mathrm{min}$ with a constant respiratory frequency of 3.3 Hz, the glomerular sensitivity increased as the airflow speed increased. $\mathrm{EC}_{50}$ values for $\mathrm{EG}$ response in the Ea glomerulus were $16 \mathrm{nM}$ at $25 \mathrm{ml} / \mathrm{min}, 5.9 \mathrm{nM}$ at $50 \mathrm{ml} / \mathrm{min}$ and $2.1 \mathrm{nM}$ at 100 $\mathrm{ml} / \mathrm{min}$. Glomerular response was not evoked in the absence of odorant stimulation at any of the airflow rates (Fig. $4 \mathrm{~A}$ ). The flow rate effects were also observed in other EG responsive glomeruli including the Eb (MOR224-5, Oka et al., 2006) and mOR-EG (MOR174-9, Oka et al., 2006) glomeruli (Fig. 4B). For both glomeruli, changes in the airflow rate from slow to fast conditions resulted in an increase in the apparent glomerular sensitivity to EG by as much as tenfold. In other words, at the sniffing state $(100 \mathrm{ml} / \mathrm{min})$, glomeruli are able to respond to a tenfold lower concentration in the air phase than normal breathing state $(25$ $\mathrm{ml} / \mathrm{min}$ ). This difference corresponds to an $\sim 100$-fold lower concentration in a solution phase (Fig. 4; also supplemental Fig. S1, available at www.jneurosci.org as supplemental material). Similar effects were also observed using the Synapto-pHluorin transgenic mice in which exocytosis from axon terminal of OSNs can be visualized (Bozza et al., 2004) (supplemental Fig. S2, available at www.jneurosci.org as supplemental material). These results demonstrated that the temporal velocity of airflow may determine the input and output sensitivity of OB. 
A
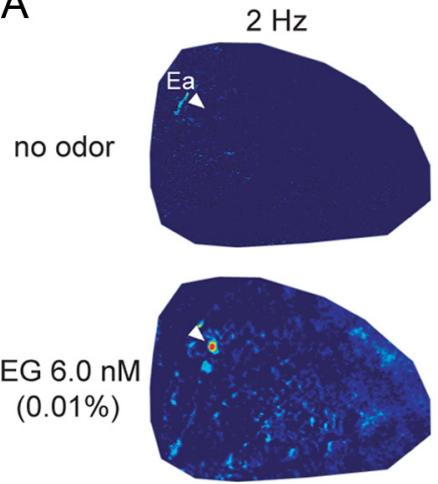

$3.3 \mathrm{~Hz}$
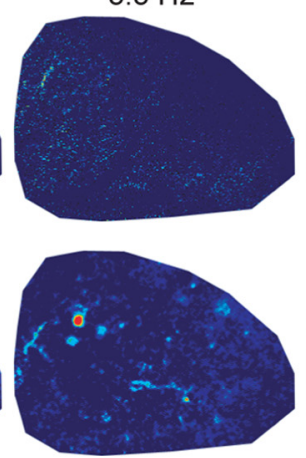
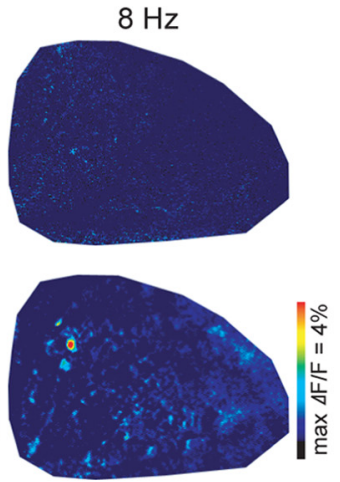

B

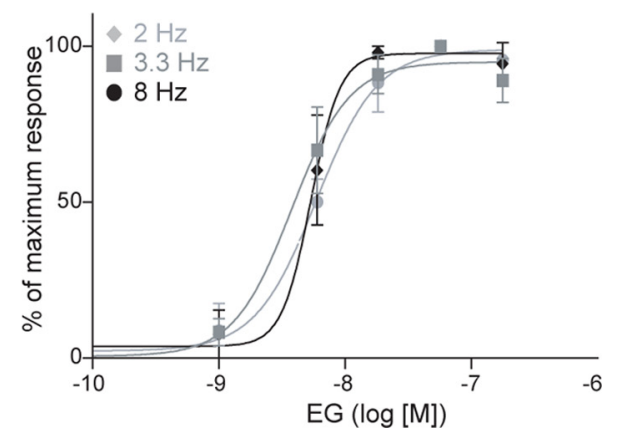

Figure 3. Effects of sniffing frequency on glomerular response sensitivity. $\mathrm{Ca}^{2+}$ response was monitored under different sniffing rates with a fixed airflow rate ( $\left.50 \mathrm{ml} / \mathrm{min}\right) . \boldsymbol{A}$, The change in fluorescence is shown in the pseudocolored images. No glomerular response was found in the absence of odorant stimulation at all frequencies. These images were obtained from the same mouse. Arrowhead indicates the position of Ea glomerulus. $\boldsymbol{B}$, The response to each concentration of $\mathrm{EG}$ in the Ea glomerulus from different animals was quantified. The results are shown as percentages of the maximum response and are fitted to the dose-response curve ( $n=3$, mean \pm SE). Each concentration point represents $0.001,0.01,0.1,1$, and $10 \%$ dilution in mineral oil.

A
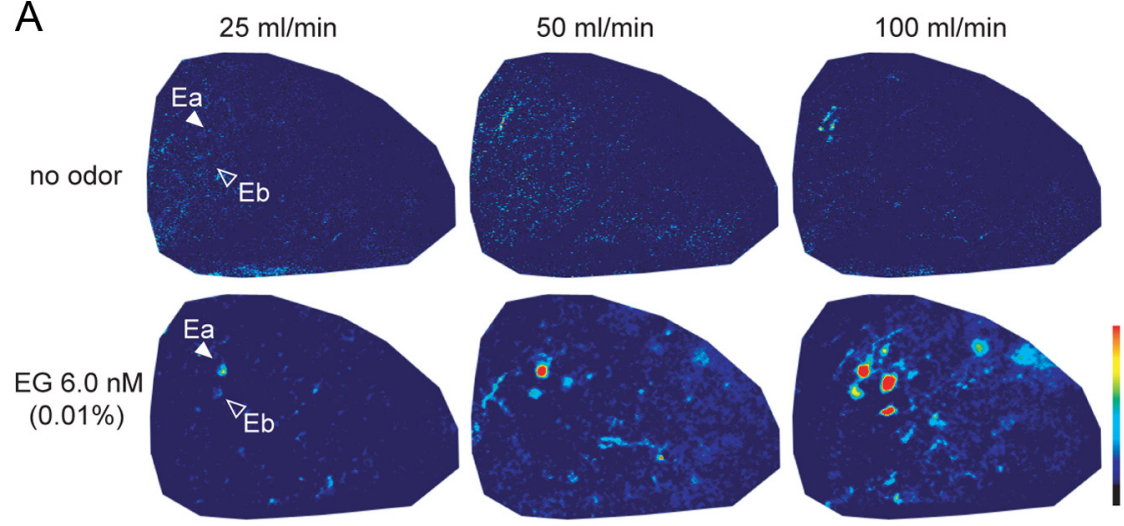

B
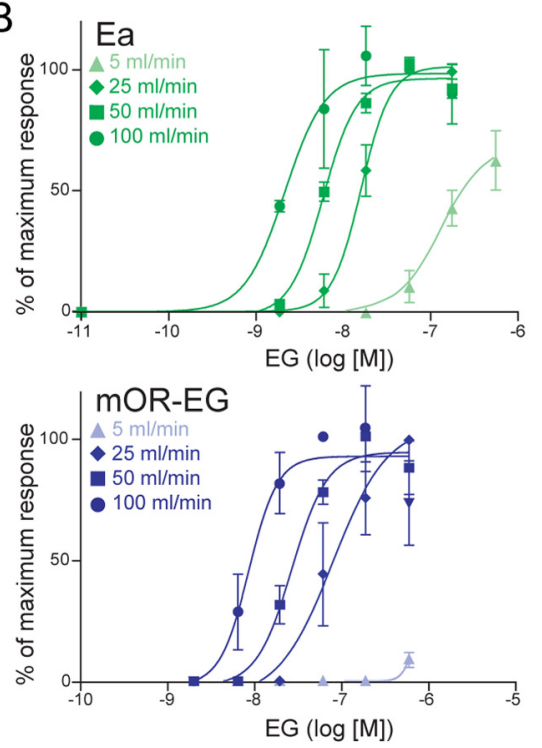

Figure 4. Effects of nasal airflow rate on glomerular $\mathrm{Ca}^{2+}$ response sensitivity. $\boldsymbol{A}$, Pseudocolored images of the odorant response in the absence (top) or presence (bottom) of EG from the same animal. Ea (filled arrowhead) and Eb (open arrowhead) glomeruli are shown. m0R-EG glomerulus is not shown in these images, as it is located in the region anterolateral of the $0 B$ and was out of focus ( $0 \mathrm{ka}$ et al., 2006). Red color corresponds to the greatest response. $B$, Dose-dependent curve of EG in receptor-defined glomeruli at different airflow rates. The responses to various concentrations of EG in the Ea (top left, $n=4$ for 25 and $100 \mathrm{ml} / \mathrm{min}$, $n=6$ for $50 \mathrm{ml} / \mathrm{min}, n=5$ for $5 \mathrm{ml} / \mathrm{min}$ ), Eb (top right, $n=4$ for 25 and $100 \mathrm{ml} / \mathrm{min}, n=6$ for $50 \mathrm{ml} / \mathrm{min}, n=5$ for $5 \mathrm{ml} / \mathrm{min}$ ), and mOR-EG (bottom, $n=4$ for 5,25 and $50 \mathrm{ml} / \mathrm{min}, n=3$ for $100 \mathrm{ml} / \mathrm{min}$ ) glomeruli from different animals were quantified and are presented as percentages of the maximum response and fitted to the dose-response curve. The data for Ea and Eb were obtained from wild-type mice and those for mOR-EG were obtained from mOR-EG transgenic mice (0ka etal., 2006). Each concentration point corresponds to a vapor pressure of $0.001,0.01,0.1,1$, and $10 \%$ dilution in mineral oil and pure EG. Data are mean \pm SE.
Furthermore, a decrease in airflow rate to $5 \mathrm{ml} / \mathrm{min}$, a rate far below than normal breathing level, resulted in an increase in the threshold of glomerular response to EG. Even using undiluted EG, which gives a vapor pressure of $551 \mathrm{nM}$, the glomerular response did not reach the maximum amplitude at the higher airflow rates (Fig. $4 B)$. Under these conditions, the response threshold concentrations were $\sim 100$-fold higher than those observed under the sniffing conditions ( $100 \mathrm{ml} / \mathrm{min})$. In particular, the threshold of the mOR-EG glomerulus was $>500 \mathrm{~nm}$ in the air phase, suggesting that $\mathrm{EC}_{50}$ values under these conditions are likely to be at the micromolar level. This threshold value is comparable to the sensitivity of $\mathrm{mOR}-\mathrm{EG}$ expressed in a heterologous system (46 $\mu \mathrm{M})$ or isolated olfactory neurons $(51 \mu \mathrm{M})$ (Oka et al., 2006). Although ORs demonstrate lower response affinities compared with other members of the G-protein coupled receptor superfamily (Katada et al., 2005), our results suggest that rapid nasal airflow may compensate for this low affinity by helping accumulate odorants into the nasal mucosa to activate ORs.

We next examined whether the flow rate effects were specific to EG or were a general phenomenon to all odorants. To test this hypothesis, we used methyl isoeugenol $(0.01 \%)$, benzene $(10 \%)$, propyl acetate (PA, $0.1 \%)$, benzaldehyde $(0.1 \%)$, valeric acid $(0.01 \%)$, and hexanal $(0.1 \%)$, which possess various functional groups, polarities, and molecular weights. It should be noted that these concentrations did not result in a saturated response under normal breathing conditions $(3.3 \mathrm{~Hz}$, $50 \mathrm{ml} / \mathrm{min}$, data not shown). The glomerular responses to these six odorants were monitored using $\mathrm{Ca}^{2+}$ imaging at three 
A<smiles>C/C=C/c1ccc(OC(C)(C)C)c(OC)c1</smiles>

benzene<smiles>c1ccccc1</smiles>
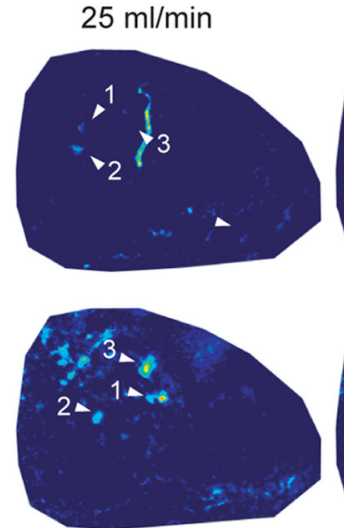

benzaldehyde<smiles>O=Cc1ccccc1</smiles>
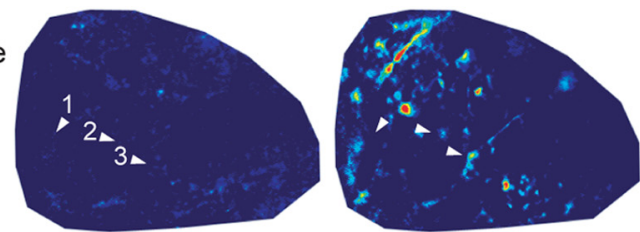

propyl acetate

(PA)<smiles>CCCOC(C)=O</smiles>
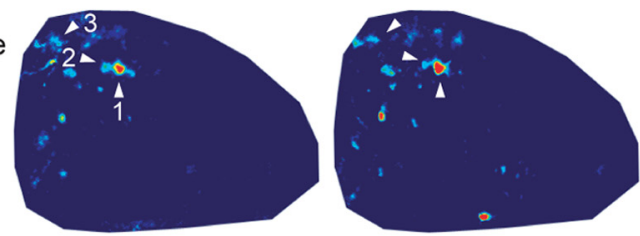

valeric acid<smiles>CCCCC(=O)O</smiles><smiles>CCCC=O</smiles>
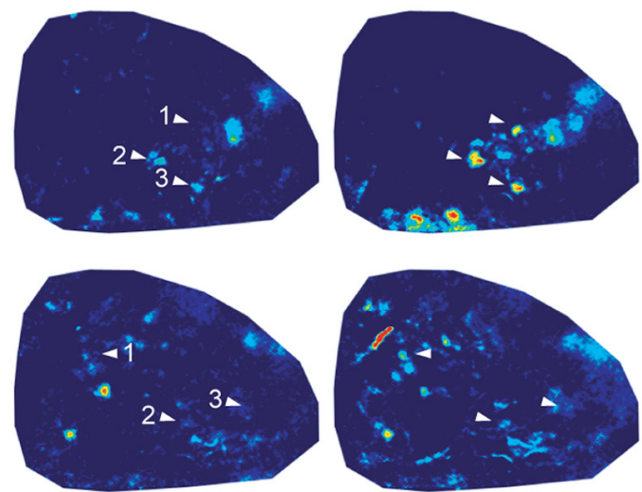

$50 \mathrm{ml} / \mathrm{min}$
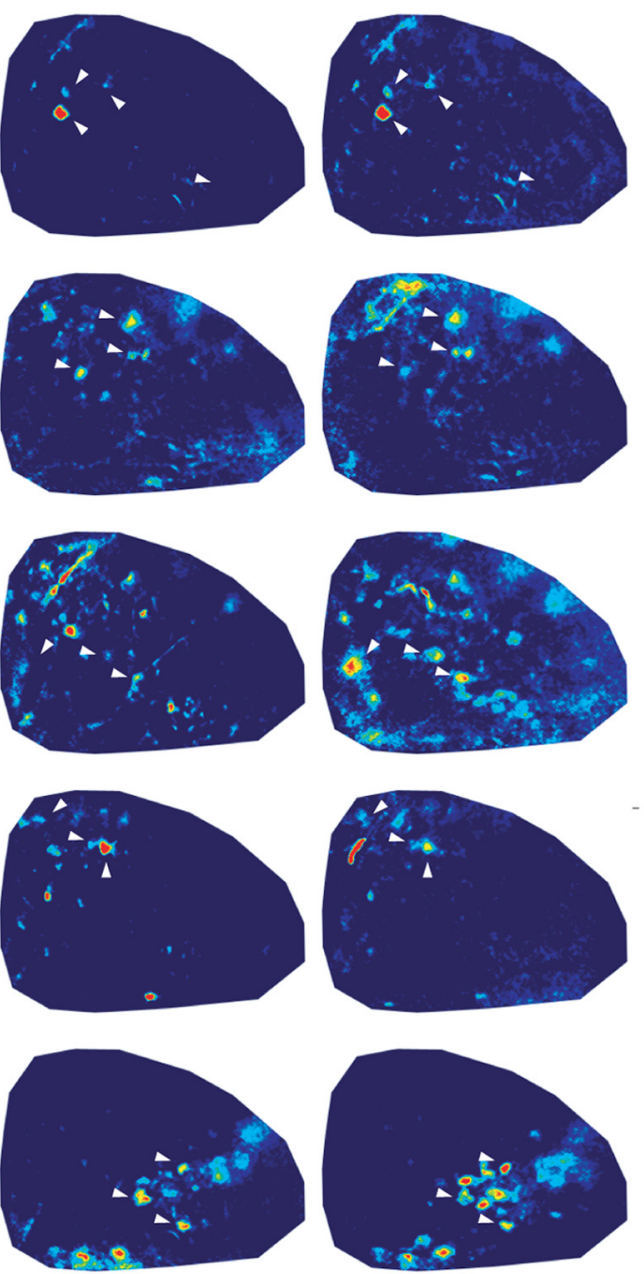

$100 \mathrm{ml} / \mathrm{min}$

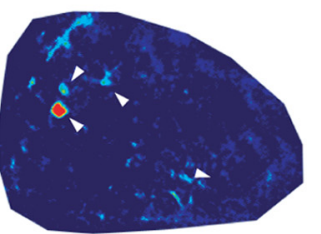

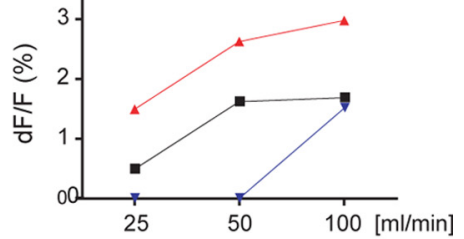
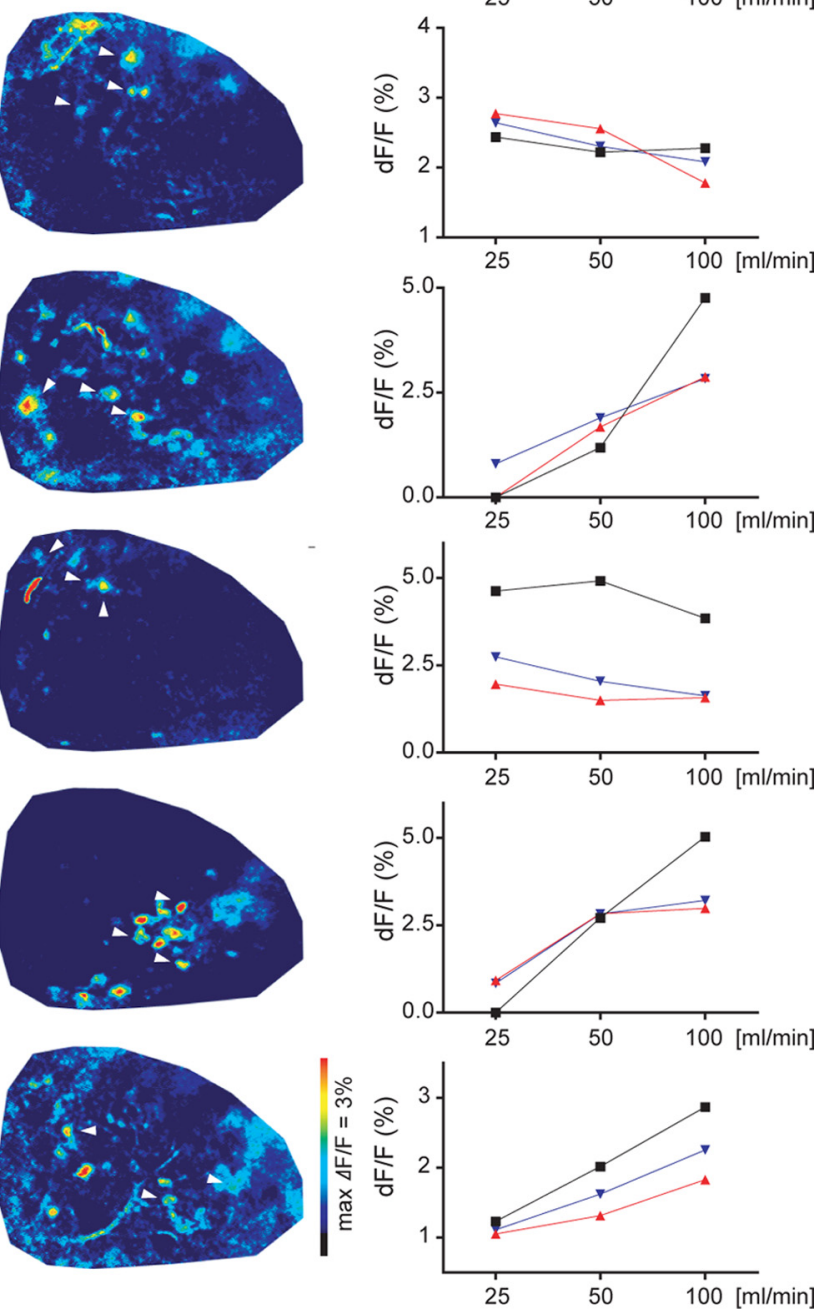

Figure 5. The variable effects of airflow rate for the different odorants. $A, 0$ dorant-evoked $\mathrm{Ca}^{2+}$ responses from six odorants are represented as pseudocolored images where red corresponds to the greatest response. Methyl isoeugenol $(0.01 \%)$, benzene $(10 \%)$, benzaldehyde $(0.1 \%), \mathrm{PA}(0.1 \%)$, valeric acid $(0.01 \%)$, and hexanal $(0.1 \%)$ were used for imaging. For each odorant, responses for different airflow rates $(25,50$, and $100 \mathrm{ml} / \mathrm{min})$ in the same animal are shown. $\boldsymbol{B}$, The response amplitude of each glomerulus at different airflow rates. Response amplitude $(\Delta F / F)$ was calculated for three glomeruli for each odorant. Each symbol corresponds to the glomerular number in $A$, where 1 is black, 2 is red, and 3 is blue.

different flow rates and at a frequency of $3.3 \mathrm{~Hz}$ (Fig. $5 \mathrm{~A}$ ). Different odorants evoked distinct and sometimes overlapping subsets of glomeruli on the dorsal surface of the $\mathrm{OB}$, representing the activation of a unique combination of ORs (Fig. 5A). Consistent with the results for EG, the sniffing frequency exhibited no effects on glomerular response to any of the odorants (data not shown). At fast airflow rate, glomeruli exhibited greater responses to methyl isoeugenol, benzaldehyde, valeric acid, and hexanal. The $\mathrm{Ca}^{2+}$ response amplitudes at $100 \mathrm{ml} / \mathrm{min}$ were 3.1 (methyl isoeugenol), 13 (benzaldehyde), 6.4 (valeric acid), and 2 (hexanal) times larger in $\Delta F / F$ compared with those at $25 \mathrm{ml} / \mathrm{min}$ (an average responses of three glomeruli for each odorant tested) (Fig. $5 B$ ). In contrast, we observed only a modest or no positive flow rate effect for PA and benzene. Responses to PA and benzene at $100 \mathrm{ml} / \mathrm{min}$ were similar or slightly lower than those at 25 $\mathrm{ml} / \mathrm{min}$ ( 0.77 and 0.75 times for benzene and PA, respectively). These results indicate that the flow rate effects greatly differ between odorants.

The next question was whether the variability in flow rate effects was due to individual odorant characteristics or the pharmacological properties of the ORs. If the former case were to be true, then the entire population of glomeruli that responds to a particular odorant should demonstrate a uniform flow rate effect. However, if it is due to OR property, then a single glomerulus should exhibit the same effect for all odorants. To distinguish these two possibilities, we looked for a glomerulus responsive to benzaldehyde and PA that showed opposite airflow rate effects (Fig. 5). In the anterior part of the dorsal OB, we identified the 
A

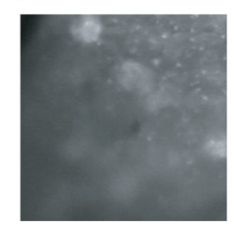

$0.1 \% \mathrm{EG}$

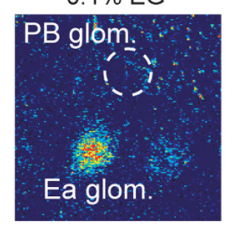

B

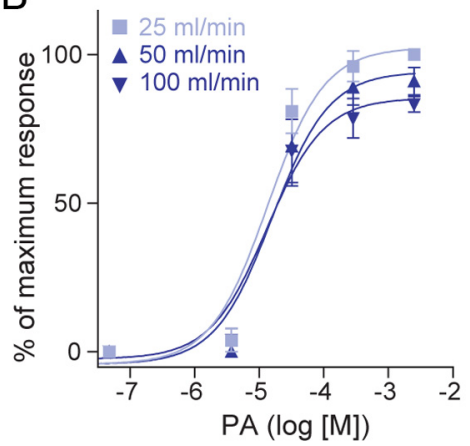

$1 \%$ PA
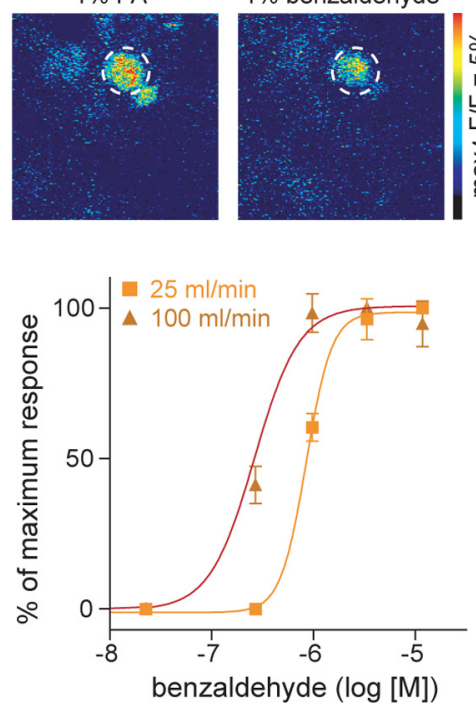

$1 \%$ benzaldehyde

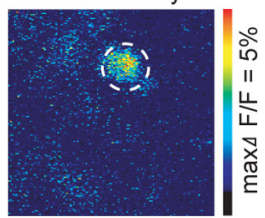

Figure 6. Different flow rate effects in a single defined glomerulus. $\boldsymbol{A}$, Location and response property of PB glomerulus. Fluorescent images (far left) and pseudocolored response images from the identical position in a single animal are shown for $0.1 \%$ EG (middle left), 1\% PA (middle right), and 1\% benzaldehyde (far right). The PB glomerulus is marked by dotted circle, which is located laterally to the Ea glomerulus that responds to both PA and benzaldehyde. $\boldsymbol{B}$, Dose-dependent response of the PB glomerulus to PA and benzaldehyde. The $\mathrm{Ca}^{2+}$ responses to different concentrations of odorants in the PB glomerulus were quantified and are presented as percentages of the maximum response and fitted to the dose-response curve ( $n=4$ for benzaldehyde, $n=3$ for PA, mean $\pm \mathrm{SE})$.

glomerulus termed the PB glomerulus, which was activated by both PA (from $0.01 \%$ ) and benzaldehyde (from $0.1 \%$ ) (Fig. 6 A). The $\mathrm{PB}$ glomerulus was positioned in a region slightly anterolateral to the EG-responsive Ea glomerulus (Fig. 6A). In the PB glomerulus, the dose- response curve of benzaldehyde was apparently shifted to the right with an increase in the airflow rate: the $\mathrm{EC}_{50}$ values for the $\mathrm{PB}$ glomerulus were $849 \mathrm{nM}$ at $25 \mathrm{ml} / \mathrm{min}$ and $284 \mathrm{nM}$ at $100 \mathrm{ml} / \mathrm{min}$ (Fig. $6 \mathrm{~B}$ ). In contrast, the dose-dependent curve for PA was similar at all nasal airflow rates. The $\mathrm{EC}_{50}$ values were $13 \mu \mathrm{M}$ at $25 \mathrm{ml} / \mathrm{min}, 15.2 \mu \mathrm{M}$ at $50 \mathrm{ml} / \mathrm{min}$, and $12.7 \mu \mathrm{M}$ at $100 \mathrm{ml} / \mathrm{min}$ (Fig. $6 \mathrm{~B}$ ). These results show that the flow rate effect predominantly depends on the odorant chemical properties but not on ORs expressed by innervated neurons.

Most natural compounds are composed of a mixture of hundreds of different odorants. For better understanding of the flow rate effects under physiological conditions, we also analyzed glomerular response to several natural flavor oils under different flow rate conditions. In the current study, we used coffee, cinnamon, rose, jasmine, peppermint, orange, and basil flavors, each of which is known to be composed of dozens of different odorants (supplemental Fig. S3, available at www.jneurosci.org as supplemental material). In the dorsal $\mathrm{OB}, \sim 50$ glomeruli were activated by each of these flavors ( $1 \%$ dilution in mineral oil). Upon comparison of glomerular response under low $(25 \mathrm{ml} / \mathrm{min})$ and high ( $100 \mathrm{ml} / \mathrm{min}$ ) airflow rates, we categorized these glomeruli into three groups: (1) glomeruli whose response was increased at 100 $\mathrm{ml} / \mathrm{min}$ by $>30 \%$ were categorized as "increased" (Fig. $7 \mathrm{~A}$, red circles), (2) glomeruli whose response was decreased by $>30 \%$ as "decreased" (gray), and (3) the remaining glomeruli were "unchanged" (blue). Interestingly, we found that the activation patterns at $100 \mathrm{ml} / \mathrm{min}$ were quite different to those observed at 25 $\mathrm{ml} / \mathrm{min}$ for all of the flavors examined (Fig. $7 A$ ). For example, the majority of glomeruli $(83 \%, 10$ out of 12 glomeruli) that responded to rose flavor exhibited a positive correlation with airflow rate, whereas responsiveness in two glomeruli were unchanged (Fig. $7 A, B$, rose). For the response to peppermint flavor, only 8 out of 20 glomeruli $(40 \%)$ showed a positive correlation with a flow rate in the same animal, while 3 and 9 glomeruli exhibited a negative and no correlation, respectively (Fig. $7 A, B$, peppermint). Thus, the positive and negative flow rate effects resulted in different response patterns in the $\mathrm{OB}$ at slow and fast airflow rates.

In contrast, an increase in concentration resulted in greater responses in most responsive glomeruli for both flavors; $10 \%$ dilution evoked responses larger than those at $1 \%$ dilution for rose ( 9 out of 10 glomeruli) and peppermint (10 out of 13 glomeruli) (Fig. 7C,D). These results indicate that flow rate effect cannot simply be argued based on the same mechanism as changing stimulus concentration. As summarized in Figure $7 B$, the flow rate effects varied between flavors likely due to the different chemical composition of the odorants (see Discussion). These data clearly show that the nasal airflow affects olfactory responses not only on single odorants but on complex mixtures of odorants, which reflects the physiological situation in the natural environment.

\section{Discussion}

Sniffing is an important behavior for vertebrates to recognize and discriminate between various odors present in the external world (Kepecs et al., 2006; Scott, 2006). The role of sniffing in olfaction has been extensively investigated using electrophysiological recordings in the OE (Mozell et al., 1991; Kent et al., 1996; Scott et al., 2006), in vivo imaging in the OB (Verhagen et al., 2007), and behavioral experiments (Laing, 1982; Kepecs et al., 2007). In the current study, we investigated two parameters involved in the sniffing behavior, sniffing frequency and airflow rate, and examined their effects on the in vivo response of glomeruli whose OR pharmacology has been well defined. To achieve this aim, we took advantage of recently developed technique that enables characterization of OR-defined glomeruli in the OB (Oka et al., 2006) and a carefully controlled artificial sniffing system. We were able to provide physiological evidence that nasal airflow rate is a key factor that regulates in vivo glomerular sensitivity to odorants. In addition, we observed both positive and negative correlations with airflow rate that were dependent on the specific chemical properties of the odorants. Our data suggest that airflow rate rather than frequency affects the temporal concentration of odorants in the nasal mucus, resulting in changes of the $\mathrm{OB}$ activation pattern between normal breathing and sniffing conditions.

In contrast to our observations, a recent study using headrestrained awake animals showed that changes in the breathing state exhibited only a minor effect on glomerular response pattern (Verhagen et al., 2007; Wesson et al., 2009). Although it is possible that two different animal states (restrained awake vs anesthetized) affected the results, we speculate that it is more likely due to concentration differences and odorant repertoires 
A
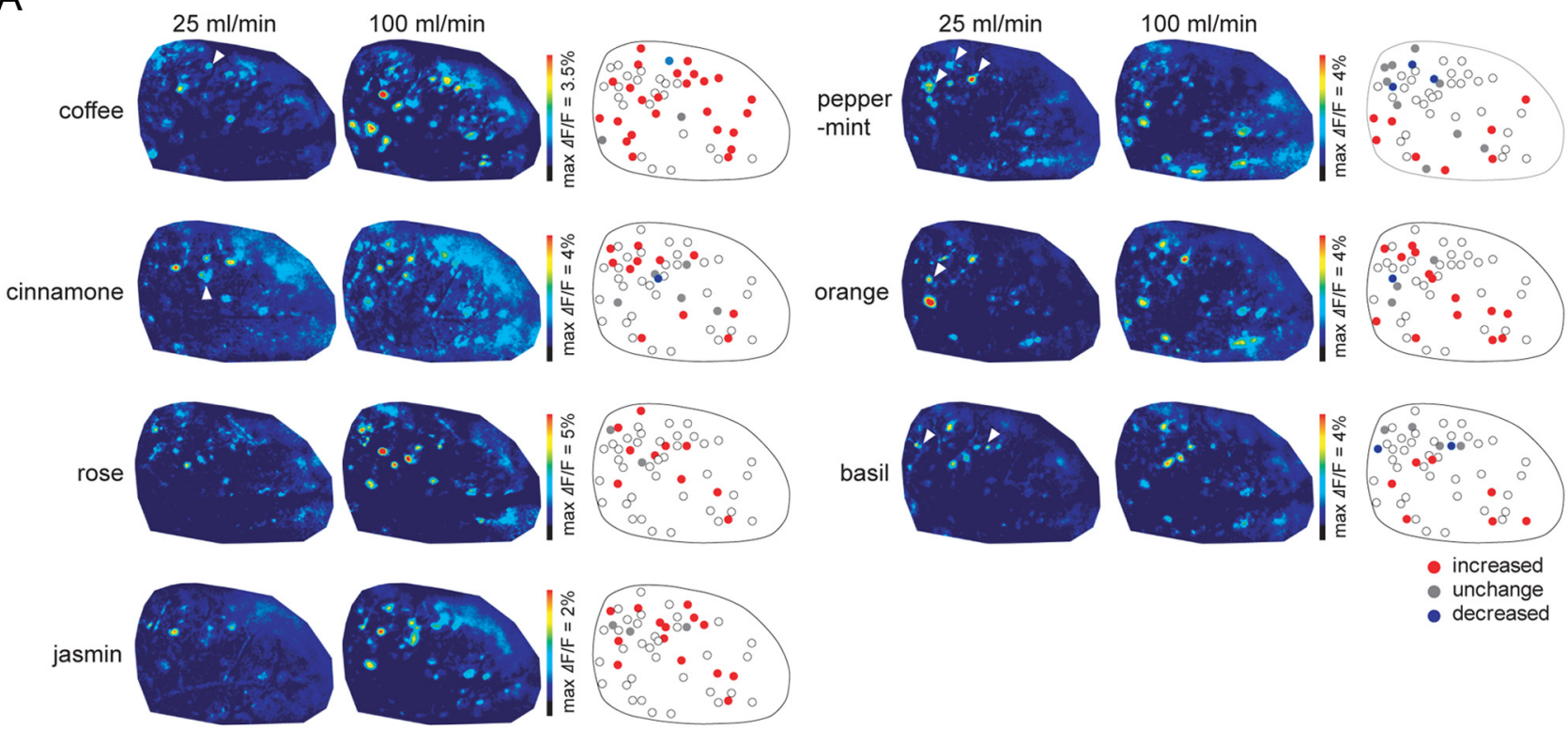

unchange

decreased

B

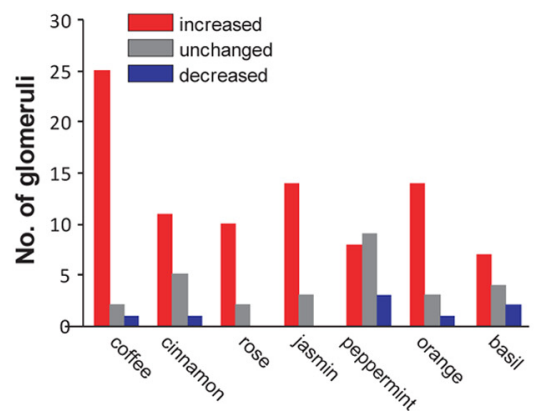

C
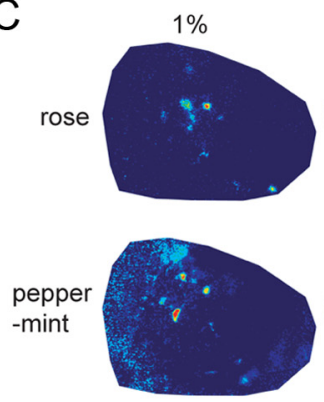
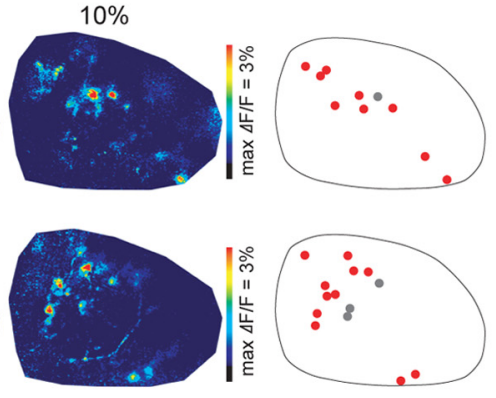

D

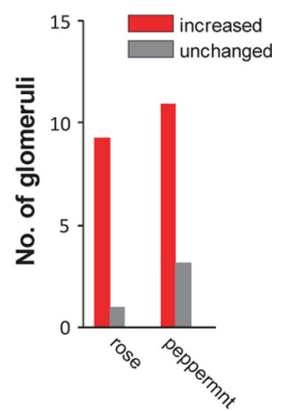

Figure 7. Glomerular responses to natural odorants at different airflow rates and odorant concentrations. $\boldsymbol{A}, 0$ dorant-evoked Ca ${ }^{2+}$ responses to seven natural flavors at 25 (left) and 100 (right) $\mathrm{ml} / \mathrm{min}$ are presented as pseudocolored images, where red corresponds to the greatest response. All pseudocolored responses were obtained from a single representative animal. Glomeruli that exhibited weaker responses at the higher airflow rates are shown by arrowheads. Right, Summary diagram of the glomerular responses. Each glomerulus was categorized into three groups that are summarized in the $\mathrm{OB}$ diagram. Glomeruli whose responses were increased by $30 \%$ at the high airflow rates were termed increased and are shown in red, and glomeruli whose responses were decreased by $>30 \%$ were termed decreased and are shown in blue, while the remainder of glomeruli were termed unchanged and are shown in gray. Glomeruli that did not respond, but did respond to other flavors, are indicated by open circles. $\boldsymbol{B}$, The number of glomeruli under the different flow rate effects. The number of activated glomeruli in $\boldsymbol{A}$ was counted and represented for each flavor. C, Glomerular responses to rose and peppermint flavors at different concentrations. Odorant responses to rose and peppermint flavor at $1 \%$ (left column) and $10 \%$ (middle column) are presented as pseudocolored images. Right, Summary diagram of the glomerular responses. Each glomerulus was categorized according to the same criteria as in $\boldsymbol{A}$. $\boldsymbol{D}$, The number of glomeruli under different airflow rates and odor concentrations for rose and peppermint flavors. The number of activated glomeruli in $C$ was counted and is represented for each flavor.

used in these experiments. In our study, the flow rate effects were observed only at lower concentrations and not for all odorants. For instance, rapid airflow rates significantly increased the response amplitude and sensitivity at $0.01 \%$ EG in most glomeruli, whereas the effect was not observed at the saturated concentrations $(>1 \%)$ (Fig. 4). Thus, an increase in nasal airflow may help detect near-threshold or subthreshold concentrations of an odorant that otherwise not be perceived under normal breathing conditions.

Although we found that airflow rate played a major role in apparent odorant sensitivity, our results do not exclude a role for sniffing frequency in odor recognition. Instead, it appears that these two factors contribute to neuronal activity in a cooperative manner. In the $\mathrm{OB}$, rapid and repeated response at sniffing has been shown to attenuate subsequent glomerular activation, which in turn enhances the contrast between sampling and background odor (Verhagen et al., 2007). In combination with our data, these results suggest that sniffing behavior may increase odor sensitivity and selectivity by altering airflow rate and frequency, respectively.
The isofunctional (lateral and medial) glomeruli located within each $\mathrm{OB}$ are reciprocally connected by interneurons that may contribute to the refinement of odorant information in the OB (Lodovichi et al., 2003). Our preliminary data regarding rheological simulation indicates that temporal and spatial distribution patterns of an odorant in the nasal cavity are quite different depending on the breathing conditions (Y. Oka, unpublished results). In light of the evidence that each glomerulus is innervated by OSNs originating from different areas of the OE, airflow rate and frequency may greatly affect the firing timing of each glomerulus and thus may affect the output signals to the higher cortex.

Under physiological conditions, the elevation of nasal airflow leads to an increase in not only odorant intake but also air pressure inside the nasal cavity, which could potentially change the odorant sensitivity. Recent in vitro electrophysiological study has shown that OSNs have the ability to respond to mechanical stimulation, suggesting that mechanical and odorant responses may be integrated in the OSN (Grosmaitre et al., 2007). We observed 
negligible mechanical response within an airflow range of this study. This was based on the following observations. (1) No specific $\mathrm{Ca}^{2+}$ response was observed during each of the sniffing cycles in the absence of odorant in this study or in other in vivo imaging experiment reported to date (Wachowiak and Cohen, 2001). (2) We found glomeruli whose responses were constant regardless of the airflow rate (Figs. 5, 6), excluding the possibility of mechanosensation-driven glomerular sensitivity shift. The mechanosensitive properties of OSNs may contribute more to the synchronization of rhythmic activity in the bulbar neurons with respiration, as suggested previously (Grosmaitre et al., 2007), rather than increase the sensitivity of the glomerulus.

In the course of examining various odorant responses, we observed a different extent of airflow effect for each odorant (Fig. 5). For example, although PA and valeric acid possess the same chemical formula $\left(\mathrm{C}_{5} \mathrm{H}_{10} \mathrm{O}_{2}\right)$, the flow rate effect was much greater for valeric acid, a result likely due to its more polar and water-soluble properties. This was also true for natural flavors. GC-MS analysis showed that the rose flavor contains a significant number and amount of polar odorants such as phenylethyl alcohol ( $\sim 65 \%$ of headspace odorants) (supplemental Fig. S3, available at www.jneurosci.org as supplemental material), whereas the peppermint flavor contained less-polar odorants as main components, including menthone ( $50 \%)$ (supplemental Fig. S3, available at www.jneurosci.org as supplemental material) (Rohloff et al., 2005). Our imaging data clearly demonstrated a greater flow rate effect for rose flavor than for the peppermint flavor (Fig. 7), showing fine correlation with the odorant polarity and flow rate effect. Our observations are consistent with those in the previous electrophysiological studies, originally demonstrated using the olfactory epithelium in amphibian species (Mozell et al., 1991; Kent et al., 1996; Scott-Johnson et al., 2000). It has been proposed that each odorant differentially diffuses inside nostril depending on their polarity and absorption rate into mucus, and consistently, the flow rate effect has been shown to correlate positively with odorant polarity. In addition, rheological modeling of the interactions between odorants and the nasal mucus layer also predicted these differences (Yang et al., 2007). Our data, together with the previous studies, suggest that mucosal absorption kinetics for each odorant presumably determines the magnitude of the flow rate effect. Further systematic analysis using a variety of odorants is required to fully explore the correlations between odorant chemical properties, mucosal solubility, and airflow effects.

It is of particular interest to ask whether the flow rate effects on glomerular responses correlated with the effect of a change in the stimulus concentration. For relatively polar odorants such as EG, an increase in airflow rate or concentration resulted in an increase in the number of responding glomeruli, indicating good correlation between the flow rate effect and the concentration effect (Fig. 4; also supplemental Fig. S4, available at www. jneurosci.org as supplemental material). In contrast, changes in odor concentration and airflow rate showed no correlation on less polar odorants such as PA (Figs. 5, 6; also supplemental Fig. S4, available at www.jneurosci.org as supplemental material). Indeed, this was also apparent for natural flavors as shown in Figure 7. Higher odor concentration evoked larger responses both for rose and peppermint (Fig. $7 C, D$ ), whereas flow rate effect varied greatly between these two flavors (Fig. $7 A, B$ ). These results indicate that changes in airflow rate and stimulus concentration differentially affect focal odor concentration in the mucus, which presumably leads to different glomerular activation pattern in the $\mathrm{OB}$.
The vertebrate olfactory system is so precise that even subtle differences in OR and glomerular activation patterns can be discriminated by the brain (Uchida and Mainen, 2003; Abraham et al., 2004; Rinberg et al., 2006; Keller and Vosshall, 2007). Using natural flavor compounds, we demonstrated that various flow rate effects for each odorant resulted in distinct glomerular activity between breathing and sniffing conditions. These results demonstrate that even a single flavor at the same concentration may be differentially represented in the $\mathrm{OB}$ under different airflow rates. It is thus provocative to address that odorants are perceived quantitatively as well as qualitatively different between these two states. In this context, we provide physiological evidence supporting the previously unexplored hypothesis that odorant representation in the $\mathrm{OB}$ is not fixed but is rather greatly variable and affected by different respiratory conditions. These results explain, in part, our experience that we are able to achieve enhanced smell ability by sniffing than under normal breathing conditions.

In conclusion, we examined the effects of airflow rate and sniffing frequency individually on apparent odorant sensitivity in the $\mathrm{OB}$ in vivo and demonstrated that nasal airflow rate is a major determinant of glomerular sensitivity. In addition, we also show that glomerular activation patterns are significantly altered by airflow rate depending on each odorant property. The current study reveals an important role for sniffing behavior and provides further clues as to how vertebrate olfaction is regulated by respiratory conditions.

\section{References}

Abaffy T, Matsunami H, Luetje CW (2006) Functional analysis of a mammalian odorant receptor subfamily. J Neurochem 97:1506-1518.

Abraham NM, Spors H, Carleton A, Margrie TW, Kuner T, Schaefer AT (2004) Maintaining accuracy at the expense of speed: stimulus similarity defines odor discrimination time in mice. Neuron 44:865-876.

Bozza T, McGann JP, Mombaerts P, Wachowiak M (2004) In vivo imaging of neuronal activity by targeted expression of a genetically encoded probe in the mouse. Neuron 42:9-21.

Buck LB (2004) Olfactory receptors and odor coding in mammals. Nutr Rev 62:S184-S188; discussion S224-S141.

Cometto-Muñiz JE, Abraham MH (2008) Human olfactory detection of homologous n-alcohols measured via concentration-response functions. Pharmacol Biochem Behav 89:279-291.

Doty RL (1994) Olfaction and multiple chemical sensitivity. Toxicol Ind Health 10:359-368.

Grosmaitre X, Santarelli LC, Tan J, Luo M, Ma M (2007) Dual functions of mammalian olfactory sensory neurons as odor detectors and mechanical sensors. Nat Neurosci 10:348-354.

Halpern BP (1983) Tasting and smelling as active, exploratory sensory processes. Am J Otolaryngol 4:246-249.

Katada S, Hirokawa T, Oka Y, Suwa M, Touhara K (2005) Structural basis for a broad but selective ligand spectrum of a mouse olfactory receptor: mapping the odorant-binding site. J Neurosci 25:1806-1815.

Keller A, Vosshall LB (2007) Influence of odorant receptor repertoire on odor perception in humans and fruit flies. Proc Natl Acad Sci U S A 104:5614-5619.

Kent PF, Mozell MM, Murphy SJ, Hornung DE (1996) The interaction of imposed and inherent olfactory mucosal activity patterns and their composite representation in a mammalian species using voltage-sensitive dyes. J Neurosci 16:345-353.

Kepecs A, Uchida N, Mainen ZF (2006) The sniff as a unit of olfactory processing. Chem Senses 31:167-179.

Kepecs A, Uchida N, Mainen ZF (2007) Rapid and precise control of sniffing during olfactory discrimination in rats. J Neurophysiol 98:205-213.

Laing DG (1982) Characterisation of human behaviour during odour perception. Perception 11:221-230.

Lodovichi C, Belluscio L, Katz LC (2003) Functional topography of connections linking mirror-symmetric maps in the mouse olfactory bulb. Neuron 38:265-276.

Mombaerts P (2004) Genes and ligands for odorant, vomeronasal and taste receptors. Nat Rev Neurosci 5:263-278. 
Mombaerts P (2006) Axonal wiring in the mouse olfactory system. Annu Rev Cell Dev Biol 22:713-737.

Mori K, Takahashi YK, Igarashi KM, Yamaguchi M (2006) Maps of odorant molecular features in the mammalian olfactory bulb. Physiol Rev 86:409-433.

Mozell M, Kent P, Murphy S (1991) The effect of flow rate upon the magnitude of the olfactory response differs for different odorants. Chem Senses 16:631-649.

Oka Y, Omura M, Kataoka H, Touhara K (2004) Olfactory receptor antagonism between odorants. EMBO J 23:120-126.

Oka Y, Katada S, Omura M, Suwa M, Yoshihara Y, Touhara K (2006) Odorant receptor map in the mouse olfactory bulb: in vivo sensitivity and specificity of receptor-defined glomeruli. Neuron 52:857-869.

Onodera M, Kuwaki T, Kumada M, Masuda Y (1997) Determination of ventilatory volume in mice by whole body plethysmography. Jpn J Physiol 47:317-326.

Rehn T (1978) Perceived odor intensity as a function of air flow through the nose. Sens Processes 2:198-205.

Rinberg D, Koulakov A, Gelperin A (2006) Speed-accuracy tradeoff in olfaction. Neuron 51:351-358.

Rohloff J, Dragland S, Mordal R, Iversen TH (2005) Effect of harvest time and drying method on biomass production, essential oil yield, and quality of peppermint (Mentha x piperita L.). J Agric Food Chem 53:4143-4148.

Scott JW (2006) Sniffing and spatiotemporal coding in olfaction. Chem Senses 31:119-130.

Scott JW, Acevedo HP, Sherrill L (2006) Effects of concentration and sniff flow rate on the rat electroolfactogram. Chem Senses 31:581-593.

Scott-Johnson PE, Blakley D, Scott JW (2000) Effects of air flow on rat electroolfactogram. Chem Senses 25:761-768.

Teghtsoonian R, Teghtsoonian M (1984) Testing a perceptual constancy model for odor strength: the effects of sniff pressure and resistance to sniffing. Perception 13:743-752.

Touhara K (2002) Odor discrimination by G protein-coupled olfactory receptors. Microsc Res Tech 58:135-141.

Touhara K (2007) Deorphanizing vertebrate olfactory receptors: recent advances in odorant-response assays. Neurochem Int 51:132-139.

Touhara K, Vosshall LB (2009) Sensing odorants and pheromones with chemosensory receptors. Annu Rev Physiol 71:307-332.

Uchida N, Mainen ZF (2003) Speed and accuracy of olfactory discrimination in the rat. Nat Neurosci 6:1224-1229.

Verhagen JV, Wesson DW, Netoff TI, White JA, Wachowiak M (2007) Sniffing controls an adaptive filter of sensory input to the olfactory bulb. Nat Neurosci 10:631-639.

Wachowiak M, Cohen LB (2001) Representation of odorants by receptor neuron input to the mouse olfactory bulb. Neuron 32:723-735.

Wesson DW, Donahou TN, Johnson MO, Wachowiak M (2008) Sniffing behavior of mice during performance in odor-guided tasks. Chem Senses 33:581-596.

Wesson DW, Verhagen JV, Wachowiak M (2009) Why sniff fast? The relationship between sniff frequency, odor discrimination, and receptor neuron activation in the rat. J Neurophysiol 101:1089-1102.

Yang GC, Scherer PW, Zhao K, Mozell MM (2007) Numerical modeling of odorant uptake in the rat nasal cavity. Chem Senses 32:273-284.

Yoshikawa K, Touhara K (2009) Myr-Ric-8A enhances G\{alpha\}15mediated $\mathrm{Ca} 2+$ response of vertebrate olfactory receptors. Chem Senses 34:15-23.

Youngentob SL, Mozell MM, Sheehe PR, Hornung DE (1987) A quantitative analysis of sniffing strategies in rats performing odor detection tasks. Physiol Behav 41:59-69. 